This item was submitted to Loughborough's Research Repository by the author.

Items in Figshare are protected by copyright, with all rights reserved, unless otherwise indicated.

\title{
High quality factor cold sintered Li2MoO4BaFe12O19 composites for microwave applications
}

\section{PLEASE CITE THE PUBLISHED VERSION}

https://doi.org/10.1016/j.actamat.2018.12.057

\section{PUBLISHER}

Elsevier () Acta Materialia Inc.

VERSION

AM (Accepted Manuscript)

\section{PUBLISHER STATEMENT}

This paper was accepted for publication in the journal Acta Materialia and the definitive published version is available at https://doi.org/10.1016/j.actamat.2018.12.057

\section{LICENCE}

CC BY-NC-ND 4.0

\section{REPOSITORY RECORD}

Faouri, Sinan S., Ali Mostaed, Julian S. Dean, Dawei Wang, Derek C. Sinclair, Shiyu Zhang, William Whittow, J. C. Vardaxoglou, and lan M. Reaney. 2019. "High Quality Factor Cold Sintered Li2moo4bafe12o19 Composites for Microwave Applications”. figshare. https://hdl.handle.net/2134/36800. 


\title{
High quality factor cold sintered $\mathrm{Li}_{2} \mathrm{MoO}_{4}-$ $\mathrm{BaFe}_{12} \mathrm{O}_{19}$ composites for microwave applications
}

\author{
Sinan S. Faouri ${ }^{1 *}$, Ali Mostaed ${ }^{1}$, Julian S. Dean ${ }^{1}$, Dawei Wang ${ }^{1}$, Derek C. Sinclair ${ }^{1}$, Shiyu Zhang ${ }^{2}$, William G. \\ Whittow $^{2}$, Yiannis Vardaxoglou ${ }^{2}$ and Ian M. Reaney ${ }^{1 *}$. \\ ${ }^{1}$ Department of Engineering Materials, University of Sheffield, Sheffield, S1 3JD, UK. \\ ${ }^{2}$ School of Electronic, Electrical and Systems Engineering, Loughborough University, Loughborough, UK.
}

\begin{abstract}
Ceramics-ceramic composites in the series (1-x) $\mathrm{Li}_{2} \mathrm{MoO}_{4}-x \mathrm{BaFe}_{12} \mathrm{O}_{19}$ (LMO-BF12, $0.00 \leq x \leq 0.15$ ) have been cold sintered at $120^{\circ} \mathrm{C}$ and their structure and properties characterized. X-ray diffraction, scanning electron microscopy (SEM) and transmission electron microscopy (TEM) confirmed that compositions were dual phase and had a dense microstructure. Composites in the $x$ BF12- $(1-x)$ LMO $(0.0 \leq x \leq 0.15)$ series resonated at MW frequencies $(\sim 6 \mathrm{GHz})$ with $5.6 \leq \varepsilon_{r} \leq 5.8$ and $Q f=16,000-22,000 \mathrm{GHz}$, despite the black colour of compositions with $x>0$. The permeability of the composites was measured in the $\mathrm{X}$ band $(\sim 8 \mathrm{GHz})$ and showed an increase from $0.94(x=0.05)$ to $1.02(x=0.15)$. Finite element modelling revealed that the volume fraction of BF12 dictates the conductivity of the material, with a percolation threshold at 10 vol.\% BF12 but changes in $\varepsilon_{r}$ as a function of $x$ were readily explained using a series mixing model. In summary, these composites are considered suitable for the fabrication of dual mode or enhanced bandwidth microstrip patch antennas.
\end{abstract}

Keywords: Cold sintering process, Ceramic material, Composite, Dielectric, Finite element modeling (FEM).

\footnotetext{
${ }^{*}$ Corresponding Authors. Department of Materials Science and Engineering, University of Sheffield, Sheffield S1 3JD, UK.

E-mail addresses:

sinan_faouri@yahoo.com (S.S. Faouri)

i.m.reaney@sheffield.ac.uk (I.M. Reaney).
} 


\section{Introduction}

Microwave (MW) dielectric ceramics have gained considerable attention because of their suitability for the fabrication of substrates, resonators and filters in wireless and satellite communication technology. Microstrip patch antennas (MPAs) have gained significant usage due to their light weight, ease of fabrication and low cost [1]. The main disadvantage of MPAs is their narrow bandwidth (BW) [2] since high permittivity $\left(\varepsilon_{r}\right)$ substrates are used to shrink their dimensions to give ultra-small, high bandwidth antennas, the goal of any MW engineer. This classic paradox of increasing $\varepsilon_{r}$ at the expense of $\mathrm{BW}$ and quality factor $(Q f)$ stimulates designers to derive different methods to enhance bandwidth, such as the use of thicker substrates or parasitic patches [2]. However, thick substrates cause problems in impedance matching, while parasitic patches linearly increase the size of the antenna and complicate its design [2]. The 'holy grail' therefore is to design advanced materials [3] [4-6], composites or metamaterials [7-9] to act as dielectric substrates for MPAs which intrinsically result in larger bandwidths.

$4 \mathrm{G}$ systems operate in the frequency range $(2-8) \mathrm{GHz}$, while $5 \mathrm{G}$ systems will ultimately operate at up to $30 \mathrm{GHz}$. For low cost antennas that are used in $4 \mathrm{G}$ systems, substrates are often polymeric but their dielectric losses may prove too high for $5 \mathrm{G}$ applications. Thus, new low cost, low loss substrates may be required. $\mathrm{Al}_{2} \mathrm{O}_{3}$ has very low dielectric loss/high quality factor $(Q f>100,000)$ and suitable $\varepsilon_{r}(10)$ but it is expensive to produce with a high sintering temperature $\left(>1500{ }^{\circ} \mathrm{C}\right)$ and is difficult to directly integrate [10]. $\mathrm{Al}_{2} \mathrm{O}_{3}$ antenna chips are fabricated separately and mechanically attached as a standalone component. Ideally, low loss/high $Q f$ ceramics are required which can be directly integrated into existing polymeric circuit boards, creating bespoke higher $\varepsilon_{r}$, high $Q f$ patches for MW antennas and filters. This would require that a MW ceramic has similar densification temperatures to that of the supporting polymer circuit board. 
Ceramics such as $\mathrm{Li}_{2} \mathrm{MoO}_{4}$ (LMO) have recently been shown by Kahari et al. [11] to densify at $120-150{ }^{\circ} \mathrm{C}$ and thus may prove useful as replacements for polymers where loss is critical. LMO has suitable MW properties for MPAs with $\varepsilon_{r}$ of $\sim(5)$, and high $Q f(30,000$ GHz) [12]. Guo et al. [13] and Wang et al. [14] have demonstrated that cold sintering not only facilitates low temperature densification but also permits the fabrication of composites, hitherto impossible by conventional sintering due to the formation of unwanted parasitic phases at high temperature deleterious to properties [15-17]. Therefore, potential new substrate design space is available through cold sintering by the formation of composites with enhanced properties; the most attractive of which from an antenna perspective would be the inclusion of a magnetic phase to increase permeability, $\mu$.

$\mathrm{Ba}$ hexaferrite, $\mathrm{BaFe}_{12} \mathrm{O}_{19}(\mathrm{BF} 12)$ is the most widely used material as a permanent magnet in the electroceramic industry, especially in magnetic storage systems, microwave devices and electromagnetic shielding due to its high Curie temperature and low cost $[18,19]$. As with all magnetic materials, the permeability of BF12 is frequency dependent from $\mathrm{MHz}$ to $10 \mathrm{GHz}$ [20] but it is known to resonate with $\varepsilon_{r} \sim 25$ at MW frequencies albeit with a low $Q f$ (2000 GHz, as measured in our study) [20-22]. It is therefore, an ideal end member in a composite ceramic to enhance the MW response of potential antenna substrates. In this contribution therefore, we demonstrate the ability to form dense $x$ BF12-(1-x) LMO composites. We address the mechanism of densification via cold sintering and illustrate how both $\varepsilon_{r}$ and $\mu$ are modified as a function of $x$. The increase in $\varepsilon_{r}$ and conductivity as a function of $x$ is further highlighted through application of finite element analysis to the composite microstructure. 


\section{Experimental}

\subsection{Synthesis}

Different proportions of BF12 (average grain size of $\sim 2 \mu \mathrm{m}$ ) and LMO (average grain size of $\sim 10 \mu \mathrm{m}$ ) were weighed and mixed for 24 hours using a variable speed ball mill. The resulting powders were sieved and mixed again using a pestle and mortar with $10-20$ wt.\% water. The wet powder was placed in either a $30 \times 30 \mathrm{~mm}$ rectangular or $20 \mathrm{~mm}$ diameter cylindrical die applying $55-70 \mathrm{MPa}$ pressure at $120{ }^{\circ} \mathrm{C}$ for $10-40$ mins to achieve densification. The pellets were left in a dry furnace at $120{ }^{\circ} \mathrm{C}$ for 24 hours. The density of synthesised pellets calculated based on the geometry of the pellets and compared to theoretical values are listed in Table 1 .

Table 1. Density $\left(\mathrm{g} / \mathrm{cm}^{3}\right)$ of the cold sintered $x$ BF12-(1-x)LMO samples.

\begin{tabular}{c|c|c|c}
\hline \hline $\boldsymbol{x}$ & Experimental (EX) & Theoretical (TH) & EX/TH (\%) \\
\hline \hline 0.05 & $2.91 \pm 0.02$ & 3.08 & $94 \pm 2$ \\
0.10 & $3.08 \pm 0.02$ & 3.13 & $97 \pm 2$ \\
0.15 & $3.02 \pm 0.02$ & 3.19 & $95 \pm 2$ \\
\hline \hline
\end{tabular}

\subsection{Structural and Microstructural Characterisation}

Crushed pellets of $x \mathrm{BF} 12-(1-x)$-LMO composites were analysed using a Bruker D2 Phaser $\mathrm{X}$-ray diffractometer in the range, $10-80^{\circ} 2 \theta$ at a scanning interval of $0.02^{\circ}$. Scanning electron microscopy (SEM) and energy dispersive X-ray spectroscopy (EDS) was carried out using a Japan Electron Optics Ltd (JEOL) Inspect $\mathrm{F}$ operating at $15 \mathrm{kV}$ equipped with an energy dispersive X-ray detector. Conventional transmission electron microscopy (TEM) was performed on an EM420 operating at $120 \mathrm{kV}$ while high resolution transmission electron microscopy (HRTEM) was performed on JEOLR005 operating at $300 \mathrm{kV}$. TEM specimens 
were pre-thinned to a thickness of $20-50 \mu \mathrm{m}$ by mechanical grinding in acetone and then ion milled to electron transparency using a GATAN precision ion polishing system (PIPS II).

\subsection{Electrical Characterisation}

Microwave dielectric properties of $x \mathrm{BF} 12-(1-x) \mathrm{LMO}$ sintered pellets were measured using a vector network analyser in a $20 \mathrm{~mm}$ diameter cavity with a current spacer of $4.87 \mathrm{~mm} . \varepsilon_{r}$ was calculated based on the dimensions and resonant frequency $\left(f_{o}\right)$ and the quality factor $(Q f)$ was determined from the width of resonant peak $3 \mathrm{~dB}$ below its maximum height. $x$ BF12-(1-x) LMO samples were also polished to match dimensions of a waveguide $(22.86 \mathrm{~mm} \times 10.16 \mathrm{~mm}$ with a thickness $<2 \mathrm{~mm})$ and measured in the $\mathrm{X}$ band. Permeability $(\mu)$ was extracted using the Nicolson-Ross-Weir technique. Impedance spectroscopy (IS) was carried out on silver coated samples in a furnace connected to E4980A Precision LCR Meter, (20 $\mathrm{Hz}$ to $2 \mathrm{MHz}$ ) at $0.1 \mathrm{~V}$ and from room temperature to $400^{\circ} \mathrm{C}$.

\section{Results}

\subsection{Structure and Microstructure}

Figure 1 shows exemplar optical images of cold sintered $x$ BF12-(1-x)LMO composites in which the black colour arises due to the presence of the ferrimagnetic BF12 phase. XRD traces of cold sintered $x \mathrm{BF} 12-(1-x) \mathrm{LMO}$ are shown in Figure 2. Each trace depicts two structures, BF12 and LMO, with no evidence of parasitic phases arising from interfacial reactions. The relative intensities of the BF12 peaks increase with $x$. SEM images of $x \mathrm{BF} 12-$ $(1-x)$ LMO composites as a function of $x$ are shown in Figure 3. The microstructure exhibits a two phase mix (as confirmed by EDX spectra, Figure 4) with smaller BF12 surrounding larger LMO grains and little porosity. Consistent with the XRD results, no secondary phases were observed in the SEM images. 


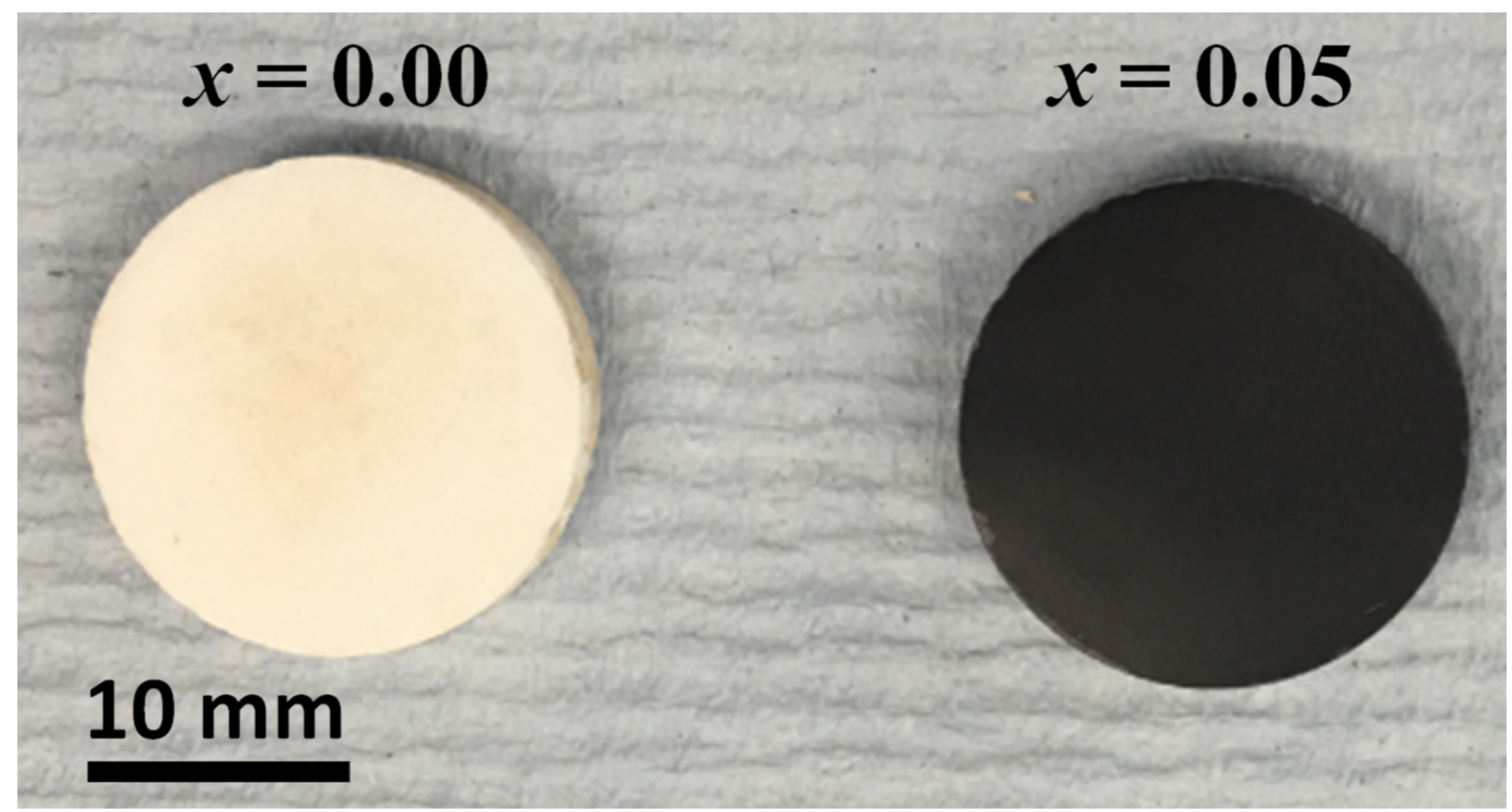

Fig. 1. Exemplar optical images of cold sintered $x \mathrm{BF} 12-(1-x) \mathrm{LMO}$ samples.

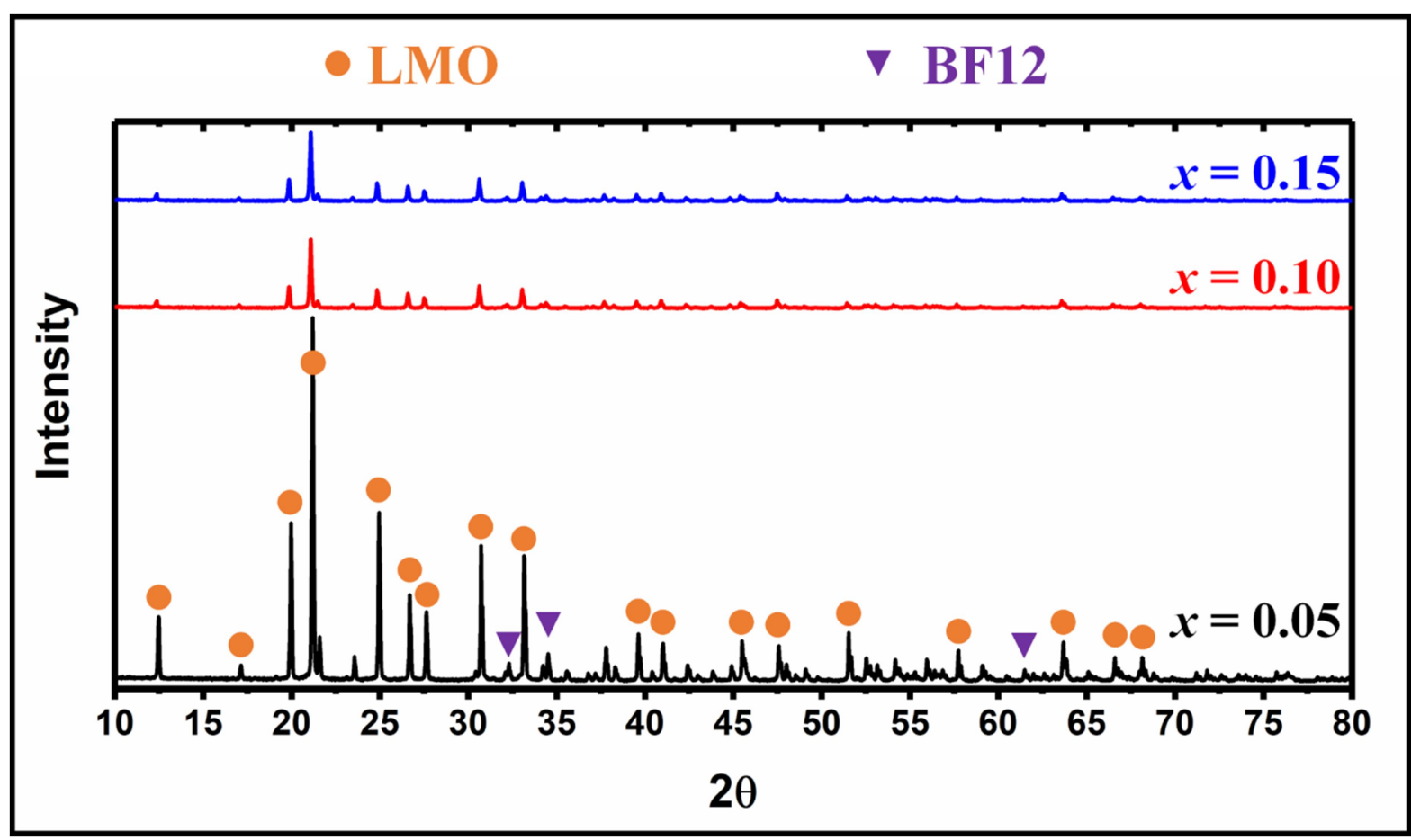

Fig. 2. XRD of cold sintered $x$ BF12-(1-x)LMO samples. 


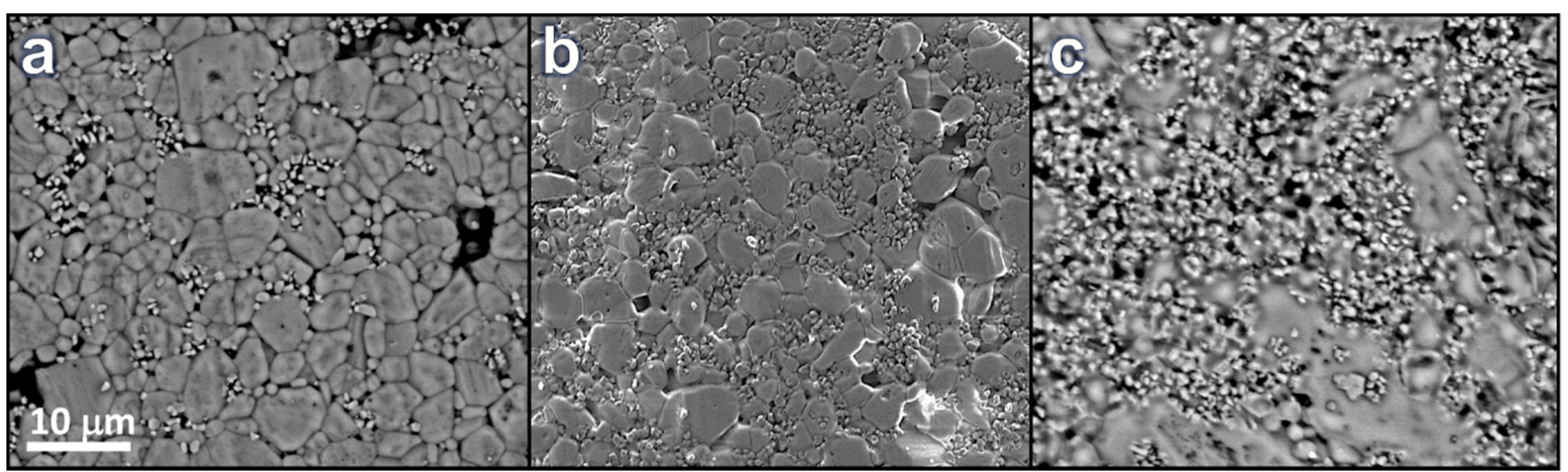

Fig. 3. SEM images obtained from cold sintered (a) 0.05BF12-0.95LMO, (b) 0.1BF120.9LMO and (c) 0.15BF12-0.85LMO samples.

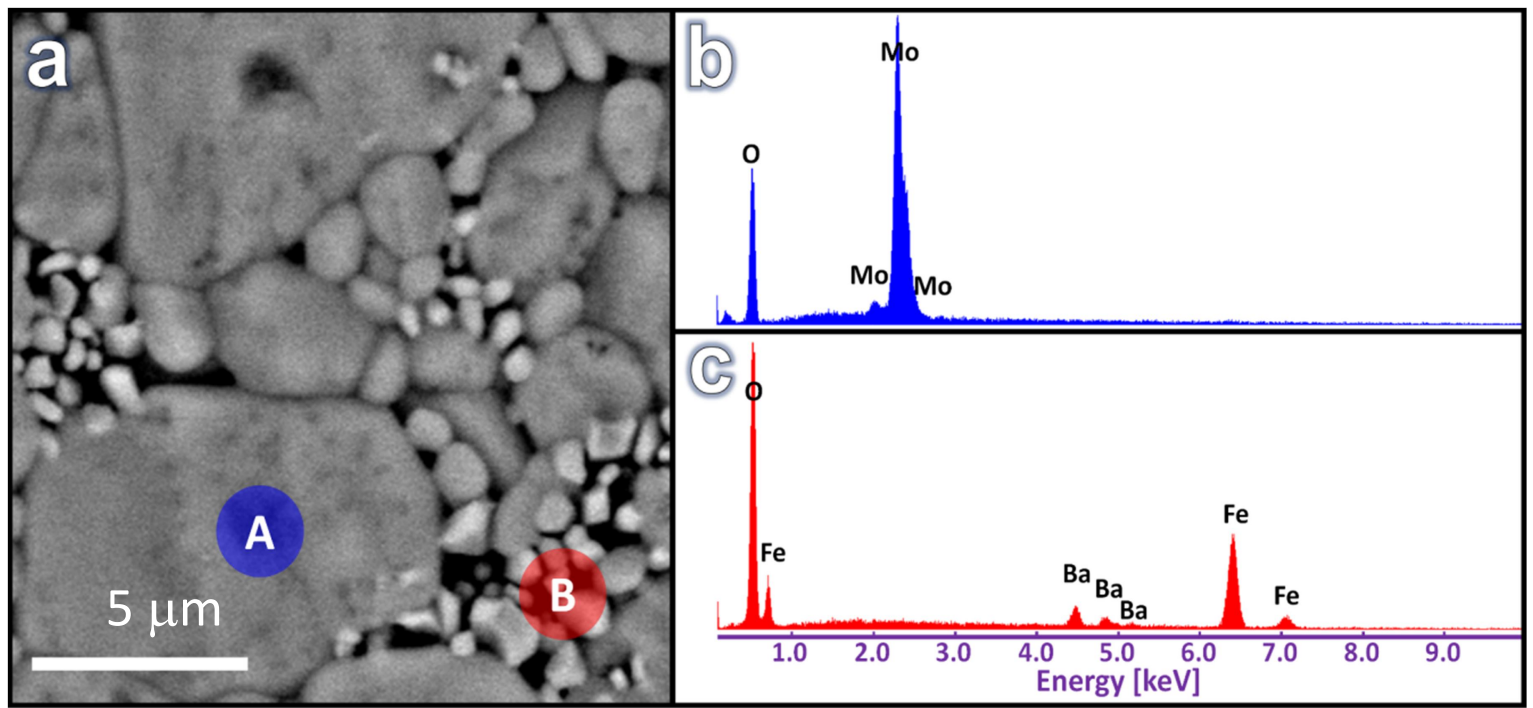

Fig. 4. (a) SEM image of obtained from the adjacent two phases in cold sintered 0.05BF120.95LMO sample (scale bar is $5 \mu \mathrm{m}$ ). (b) and (c) show representative energy dispersive X-ray spectra acquired from the large grains (area marked by "A" in (a)) and the small grains (area marked by "B" in (a)), respectively.

Figures 5 (a) and (b) are TEM images of grains of LMO and BF12 in cold sintered 0.15BF12-0.85LMO, respectively. Inset in Figures 5 (a) and (b) are [010] and [100] zone axis diffraction patterns from LMO and BF12, respectively which conclusivley identify each phase. The TEM images confirm the two phase mix identified by XRD and SEM. In addition, HRTEM images from the surface of BF12 particles (e.g. Figures 5(c-e)) reveal that LMO surrounds each grain of BF12. The mechanism of densification therefore, relies on the 
dissolution of LMO grains into the added water which under a combination of pressure and capillary action surrounds BF12 grains. The BF12 grains themselves do not undergo significant dissolution. Simultaneously, particle rearrangement and enhanced packing occur under pressure in the die. As water evaporates, $\mathrm{Li}^{+}$and $\left(\mathrm{MoO}_{4}\right)^{-}$ions crystallise on the surface of the LMO and BF12 grains, thus densifying not only clusters of LMO particles but also regions of BF12. The net result is a dense composite with limited interaction between the end member phases. A schematic of this mechanism is shown in Figure 6.

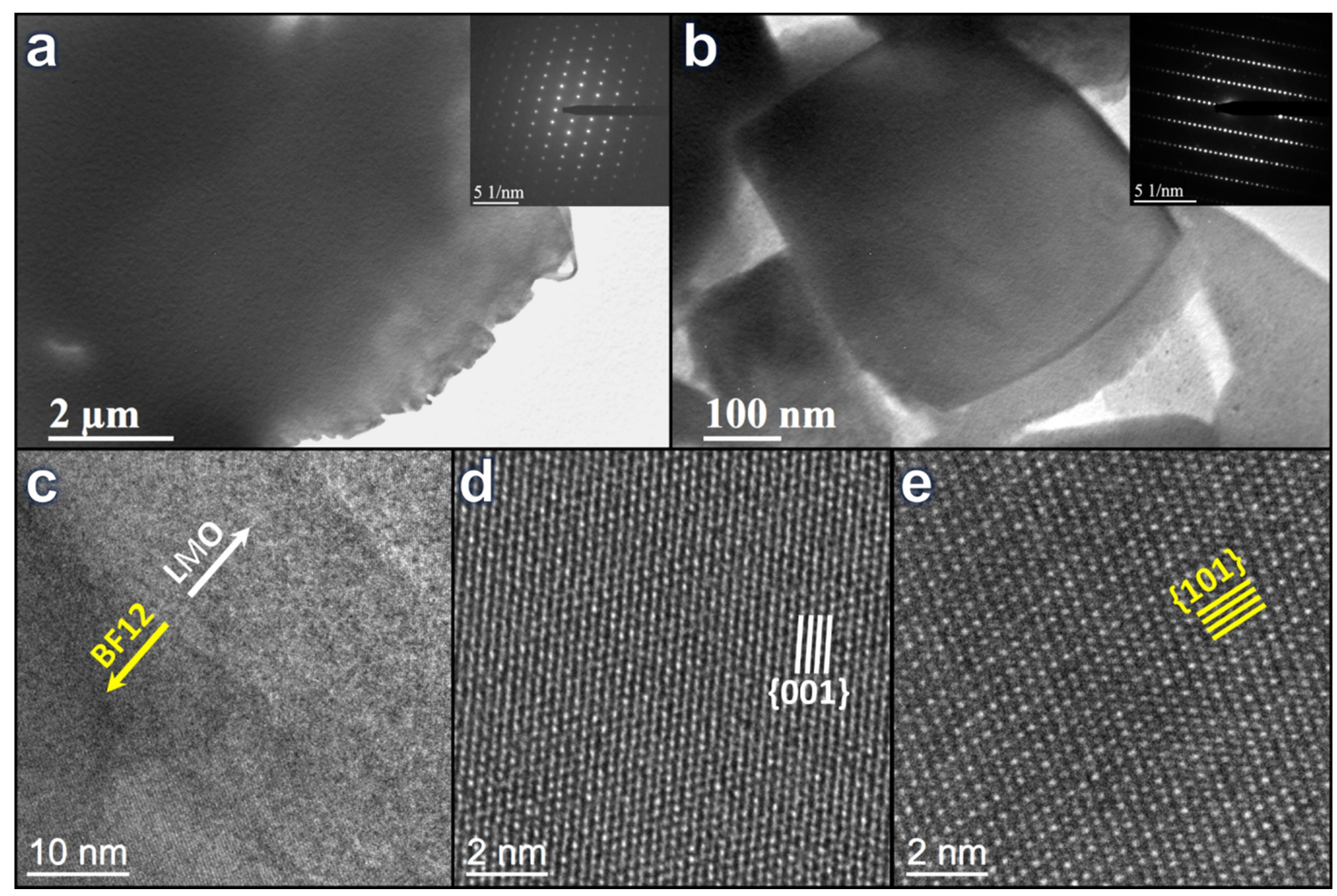

Fig.5 TEM image from cold sintered 0.15BF12-0.85LMO showing (a) a LMO grain; inset is the 010 zone axis diffraction pattern of the LMO grain. (b) a BF12 grain; inset is the 100 zone axis diffraction pattern of the BF12 grain. (c) An HRTEM image obtained from the interface of a BF12 and LMO grains. (d) and (e) show HRTEM images at a higher magnification from the LMO and BF12 sides of the interface shown in (c), respectively. Note that LMO surrounds the BF12 grains. 


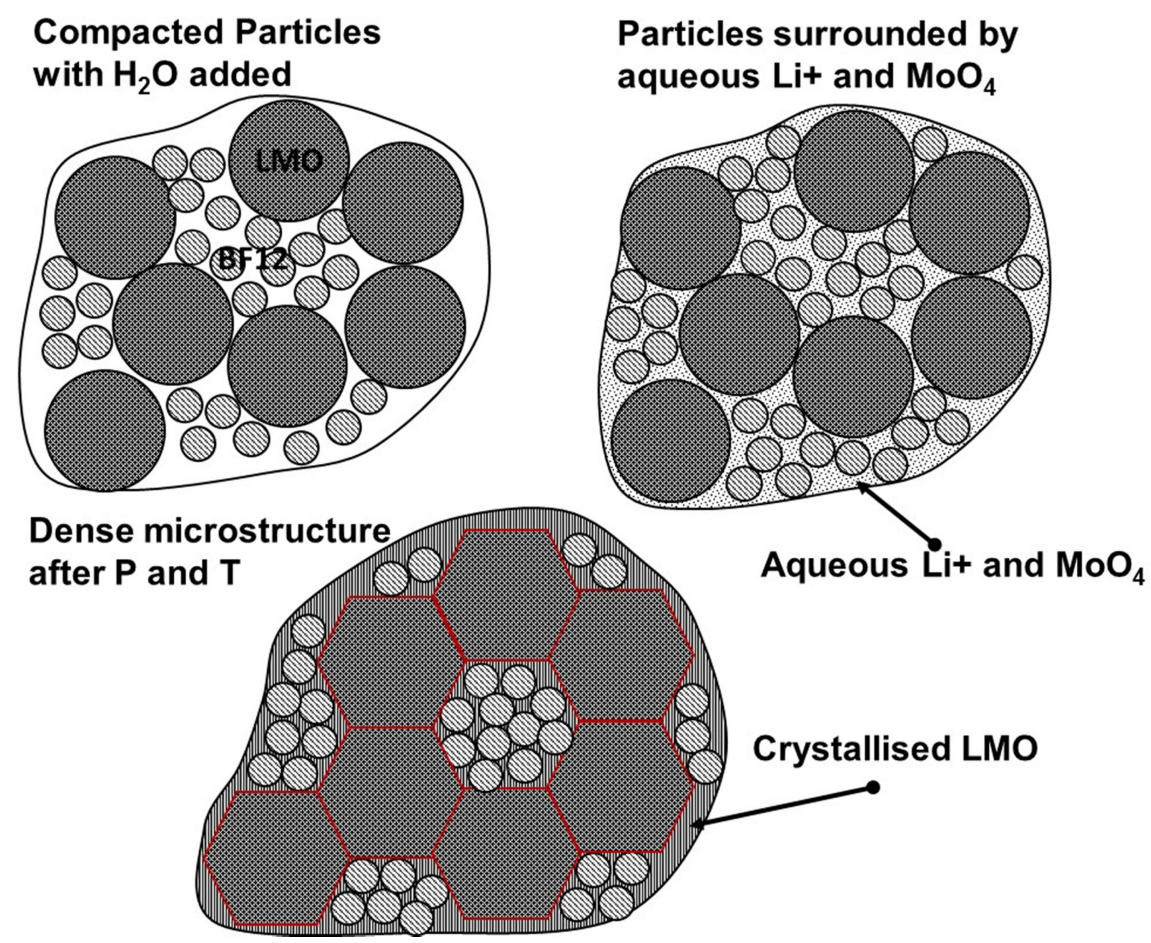

Fig. 6. Schematic representation of the densification of LMO-BF12 composites at $120{ }^{\circ} \mathrm{C}$.

\subsection{Electrical Properties}

Figure 7(a) shows $\varepsilon_{\mathrm{r}}$ (black line) and $Q f_{o}$ (blue line) as a function of $x$. $\varepsilon_{r}$ increased with inceasing BF12 concentration but $Q f_{o}$ decreased from around $24,000 \mathrm{GHz}$ to $17,000 \mathrm{GHz}$. These results are consistent with BF12 having a higher $\varepsilon_{r}(25)$ and lower $Q f(<2000 \mathrm{GHz})$ than LMO. However, the values of $Q f$ are remarkably high for a composite material and show great promise for MW applications, provided there is also an enhancement in $\mu$ from the addition of the BF12 end member. 


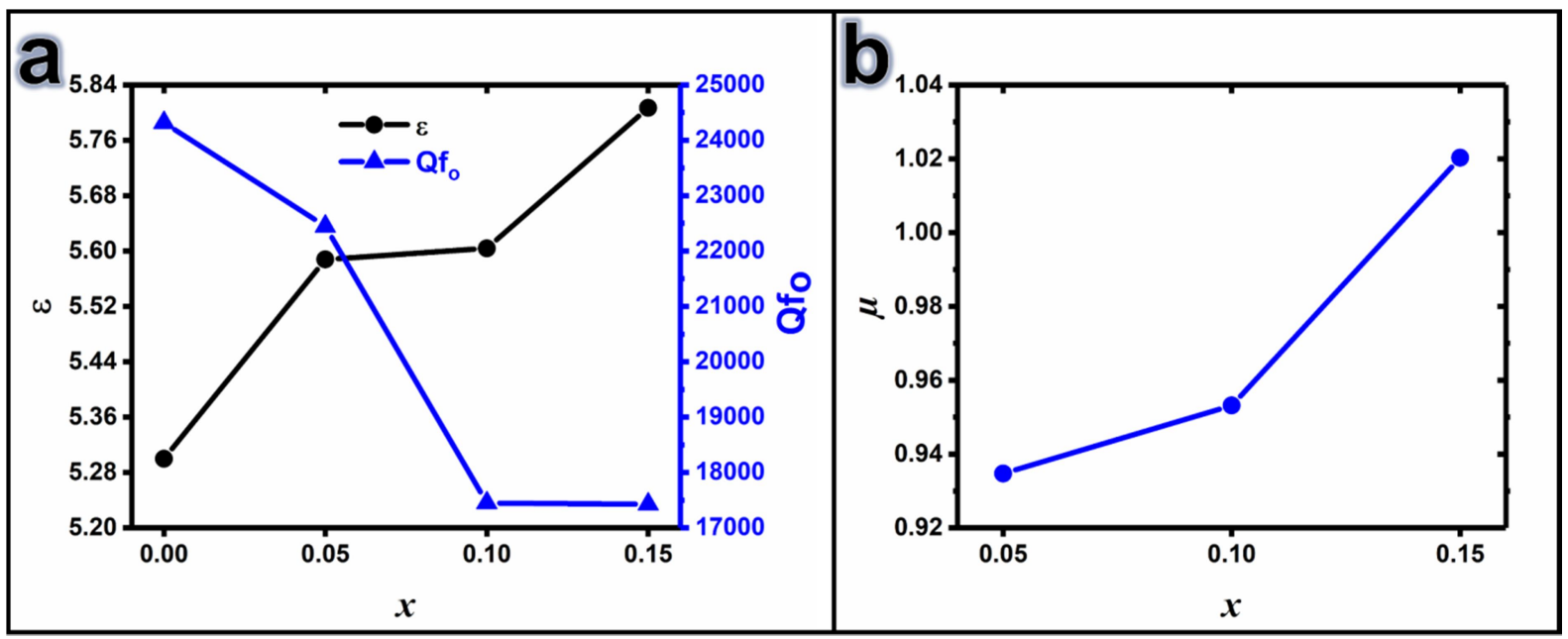

Fig. 7 (a) $\left(\varepsilon, Q f_{o}\right)$ and (b) $\mu$ at $\mathrm{X}$ band frequencies $x$ BF12-(1-x)LMO composites

Figure 7 (b) shows $\mu$ measured in the X-band ( $\sim 8 \mathrm{GHz})$ for $x$ BF12-(1-x)LMO composites. $\mu$ increases with the volume fraction of BF12 from $\sim 0.93$ at $x=0.05$ to 1.02 at $x=0.15$, consistent with the measured values in the previous research works; e.g. Bahadoor et al. [20] showed $\mu=1$ for BF12 at frequencies from 8-26.5 GHz. Although these are comparatively low values, $\mu$ increases as frequency decreases in BF12, doped BF12 and their composites [23-27]. The increment in $\mu$ would therefore be substantial when frequency decreases to $0.5-3$ $\mathrm{GHz}$ [23-27] and would contribute significantly to the EM response. Nonetheless, the values of $\mu$ attained in the $\mathrm{X}$ band suggest a systematic increase as a function of $x$ coupled with a high $Q f$.

Figure 8 shows Arrhenius plots of bulk conductivity data obtained from spectroscopic plots of the imaginary components of the electric modulus $\left(M^{\prime \prime}\right)$ which compare cold sintered 0.1BF12-0.9LMO composites with, conventionally sintered BF12 and conventionally sintered LMO. As expected, the BF12 ceramic has much higher bulk conductivity compared to LMO. The conductivity of the composite ceramics is intermediate in magnitude compared to the end members. The values of activation energy associated with the bulk response extracted from the data reveal that cold sintered 0.1BF12-0.9LMO has an activation energy 
of $0.25 \mathrm{eV}$ which is comparable to $0.29 \mathrm{eV}$ of the more conductive end member (BF12) and is much lower than $1.28 \mathrm{eV}$ associated with conventionally sintered LMO. These data suggest that a percolation pathway exists in the composite with the current choosing the path of least resistance via the higher conducting BF12 phase.

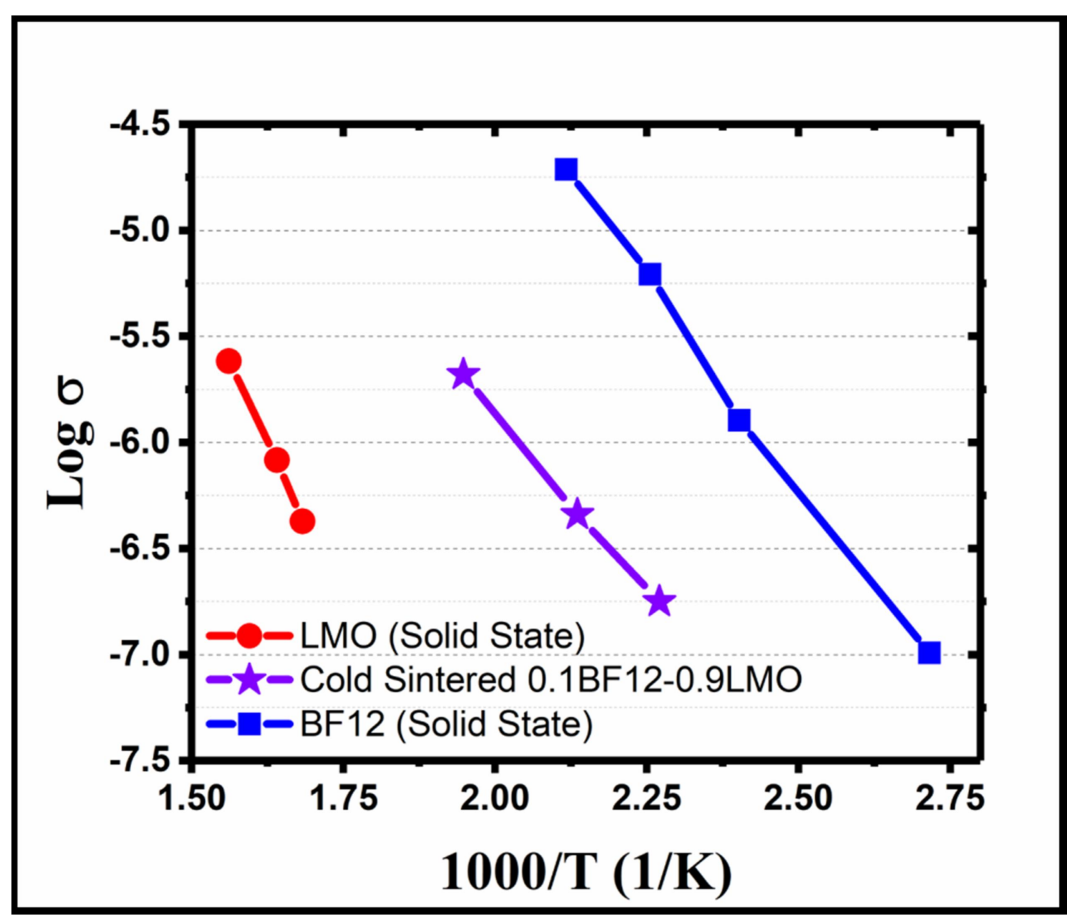

Fig. 8. Arrhenius plot of bulk conductivity of conventionally sintered ceramics of the end members with a cold sintered 0.1BF12-0.9LMO. NB: details for the fabrication of conevntionally sintered BF12 and LMO can be found in [28, 29], respectively.

\subsection{Finite Element Modelling of the composite microstructure}

Finite element modelling was used to study the $x \mathrm{BF} 12-(1-x) \mathrm{LMO}$ composite properties for various volume fractions. One challenge in replicating the composite is the simulation of a microstructure that consists of two distinct grain sizes. Adopting a Voronoi tessellation [30] is insufficient to replicate this microstructure, as the algorithm divides up the space between the initial seed points. The small grains thus grow as they near the larger grains, generating a normal distribution. Here, we use a feature in Voro++ [31] known as radical Voronoi 
tessellation. Each discretized seed point is weighted with lower and higher values to give smaller and larger BF12 and LMO grains, respectively. A binomial grain size of polydisperse particles is generated which is matched to the experimental distribution. Each of the two materials is assigned experimental values from which we extract the room temperature $\sigma$ (Figure 8) and $\varepsilon_{r}$. The values extracted for LMO gave $\sigma=2.26 \times 10^{-15} \mathrm{Scm}^{-1}$ and $\varepsilon_{r}=5.33$, compared to $\sigma=4.07 \times 10^{-8} \mathrm{Scm}^{-1}$ and $\varepsilon_{r}=25$ for BF12. Various volume fractions of LMO were then simulated, generating approximately 2000 small grains for a pure BF12 model, and over 500 large grains for LMO (Figure 9a). The structures were then meshed with between 1$2 \mathrm{M}$ tetrahedral elements and run using the in-house finite element package ElCer [30] to simulate the effective $\varepsilon_{r}$ and $\sigma[30,32]$.
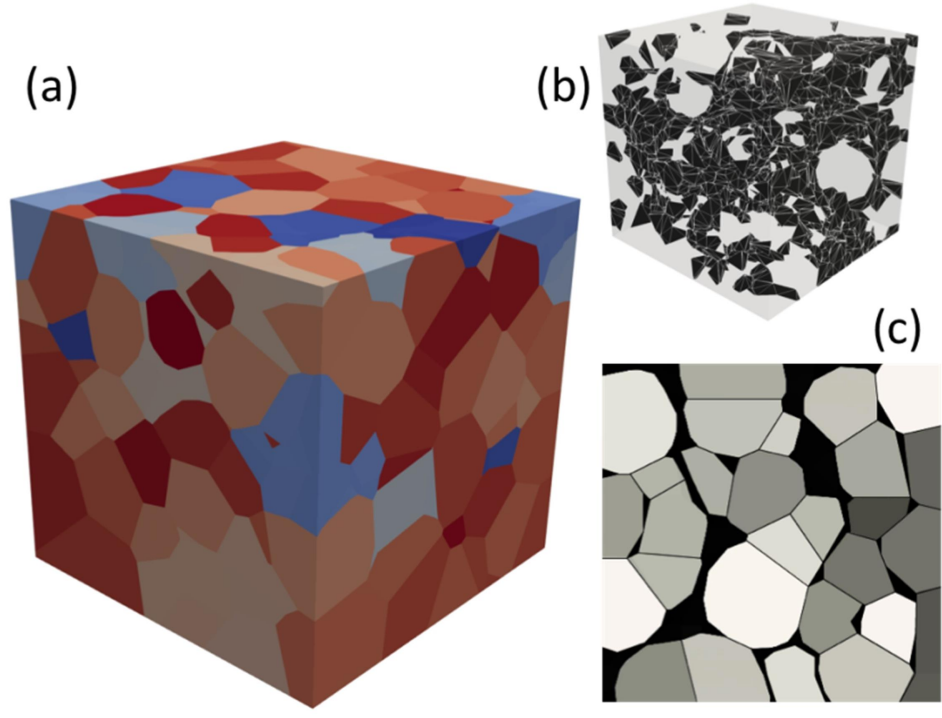

(d)

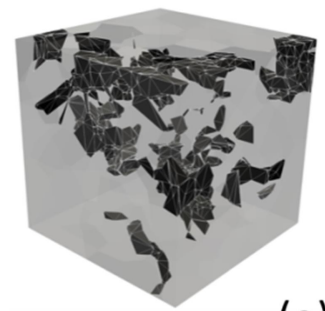

(c)

(e)
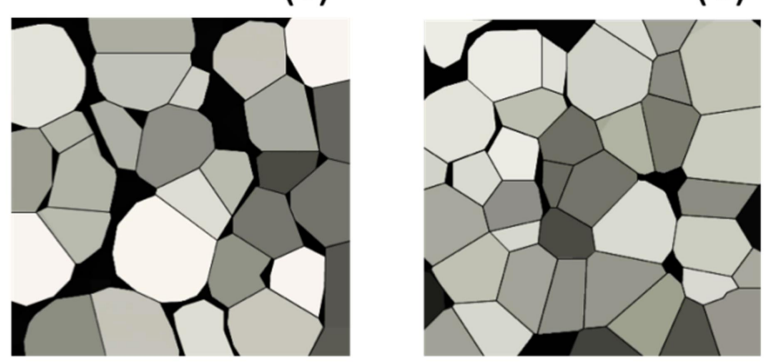

Fig. 9. (a) an example of the full 3D finite element microstructure containing between 500 1500 individual grains of an a LMO microstructure. (b) and (d) show the microstructure, by displaying the smaller more conducting BF12 grains in black. (c) and (e) are slice plots of the models where for simplicity, the black regions are designated as collections of small BF12 grains. For a volume fraction with 20\%BF12 (b and c), a continuous network of BF12 grains describes a conductive path from the top to the bottom but which is absent for 5 vol\% BF12 (d and e). 
In generating these microstructures, the volume fraction of the smaller BF12 is crucial since they surround the larger LMO grains and relatively few are needed to create a conductive network. The BF12 grains effectively by-pass the resistive LMO grains, as shown in Figures 9(b) and 8(c) for 20 vol.\% BF12. At lower values of $x$, these paths become discontinuous with gaps forming, Figures 9(d) and 8(e) for 5 vol.\%. This percolation threshold is observed directly in the measured $\sigma$. Figure 10 highlights the extracted $\sigma$ and $\varepsilon_{r}$ arising from the FEM simulations. For 5 vol.\% BF12, $\sigma$ rises but at 10 vol.\% there is a discontinuous change, attributed to the formation and conducting pathways. For $>10$ vol.\% BF12, there is gradual convergence towards $\sigma$ of the BF12 end member. However, for $\varepsilon_{r}$ no percolation effect is observed and the value tends towards LMO, consistent with a parallel summation, predicted by series mixing rules:

$$
\frac{1}{\varepsilon}=\frac{V_{B F}}{\varepsilon_{B F}}+\frac{V_{L M O}}{\varepsilon_{L M O}}
$$

where $\mathrm{V}_{\mathrm{BF}}, \mathrm{V}_{\mathrm{LMO}}, \varepsilon_{B F}, \varepsilon_{L M O}$ are the volume fractions and $\varepsilon_{r}$ of the BF and LMO phases, respectively.

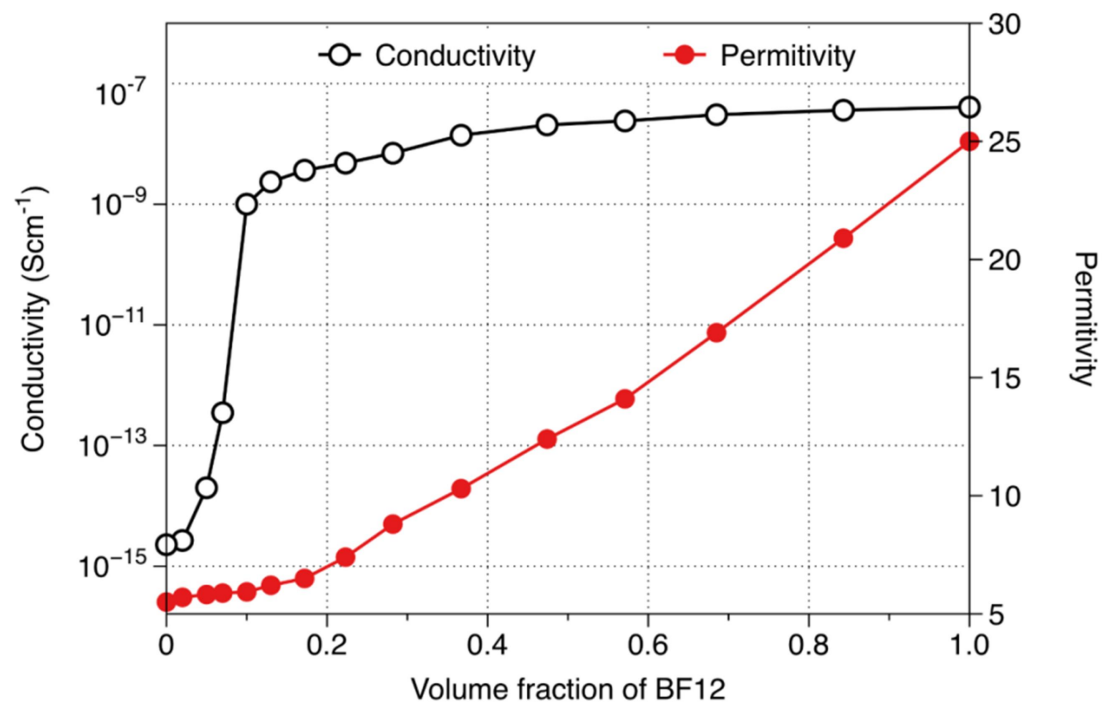

Fig. 10. The extracted conductivity $(\sigma)$ and permittivity $\left(\varepsilon_{r}\right)$ from the finite element simulations highlighting a percolation threshold for conductivity below for $x<0.1$ volume fraction. 


\section{Discussion}

Cold sintered composites of $x \mathrm{BF} 12-(1-x) \mathrm{LMO}$ offer several advantages over there conventionally sintered counterparts. Cold sintering is a more efficient processing route for the fabrication of composites, reducing the energy consumed by approximately $50 \%$ with a commensurate reduction in $\mathrm{CO}_{2}$ emissions. In addition, densification is achieved comparatively easily which is not the case for conventionally sintered composites. Most crucially, the negligible interaction between the end members results in only a limited decrease in $Q f$ despite the addition of $15 \mathrm{wt} \%$ BF12, unlike during conventional sintering where interaction causes a catastrophic deterioration in properties [33, 34]. Guo et al. [13] compared cold with conventionally sintered LMO. They reported that cold sintered LMO showed superior $\varepsilon_{r}$ (5.6 against 5.5) but lower $Q f(30,500$ against $45,000 \mathrm{GHz})$ than conventionally sintered ceramics [13]. One further advantage often overlooked, is that the lateral dimensions in cold sintering are precisely confined to the width of the die, i.e there is zero lateral shrinkage. This reduces the need for extensive post-sinter machining of parts to tune their dielectric properties. This latter advantage is important for the fabrication of low cost RF substrates and devices and could, coupled with the low densification temperature, revolutionize the manufacturing of ceramics in RF devices.

As discussed by Randall and co-workers [13], cold sintered $\left(120{ }^{\circ} \mathrm{C}\right)$ ceramics may be integrated with polymers and offer the potential through screen printing of the fabrication of layers of ceramic directly onto circuit boards, thereby enhancing $\varepsilon_{r}, \mu$ and $Q f$ of the MW substrate. Recently, Wang et al. [14] reported temperature stable cold sintered composite with $Q f \sim 7000$ and $\varepsilon_{r} \sim 17$. The direct integration of $x \mathrm{BF} 12-(1-x) \mathrm{LMO}$ and related cold sintered materials onto PCBs may offer technological solutions in devices where the dielectric loss of the substrate is paramount or potentially when a dual mode RF response is required based on $\mu$ and $\varepsilon_{r}$. 
Mechanical property measurements were not performed on these ceramics but they could be handled readily to perform all measurements. Moreover, the intended application would be as substrates integrated into PCBs in which the mechanical properties of the ceramic are less relevant than that of the module as a whole.

\section{Conclusions}

$x$ BF12-(1-x)LMO composites were cold sintered at $120{ }^{\circ} \mathrm{C}$ and their microwave properties measured. All ceramics showed a good densification with low porosity and high quality factor. TEM showed no evidence of interaction of BF12 with LMO. $\varepsilon_{r}$ increased to 5.8 for compositions with $\mathrm{x}=0.15$ with $Q f=17,000 \mathrm{GHz}$ while $\mu$ showed a systematic increase as a function of $x$. The impedance analysis accompanied by FEM suggest that for $>10$ vol.\% BF12 a percolation pathway exists in the composite with the current choosing the path of least resistance via the higher conducting BF12 phase. The combination of novel properties and easy integration into PCBs make cold sintered composites of this type attractive for future RF applications.

\section{Acknowledgments}

Ian M. Reaney and Julian S. Dean would like to acknowledge the Engineering and Physical Sciences Council (EPSRC) grants, EP/L017563/1 and EP/N010493/1.

\section{References}

[1] I. Singh, V.S. Tripathi, Micro strip patch antenna and its applications: a Survey, Int. j. comput. technol. appl. 2 (2011) 1595-1599

[2] C.L. Mak, K.M. Luk, K.E. Lee, Y.L. Chow, Experimental study of a microstrip patch antenna with an Lshaped probe, IEEE Trans. Antennas Propag. 48 (2000) 777-783

[3] A.A. Hanzaz, Development of microstrip patch antenna with change in materials and dimensions using MCM technology, 2016 5th International Conference on Electronic Devices, Systems and Applications (ICEDSA) (2016) 1-4

[4] M. Dashti, J.D. Carey, Graphene Microstrip Patch Ultrawide Band Antennas for THz Communications, Adv. Funct. Mater.28 (2018) At. 1705925 
[5] G. Kaur, E.S. Goyal, To study the effect of substrate material for microstrip patch antenna, IJETT, 36 (2016) 490-493

[6] M. Islam, R. Rashidul Hasan, M. Rahman, K. Islam, S.M. Al-Amin, Design \& Analysis of Microstrip Patch Antenna Using Different Dielectric Materials for WiMAX Communication System, iJES. 4 (2016) At. 5569

[7] C.V. Mahamuni, Performance enhancement of microstrip patch antenna using metamaterial cover, 2016 International Conference on Global Trends in Signal Processing, Information Computing and Communication (ICGTSPICC). (2016) 382-388.

[8] M. Mehrparvar, F. Hodjat Kashani, Microstrip antenna miniaturization using metamaterial structures, 20th Iranian Conference on Electrical Engineering (ICEE2012) (2012) 1243-1246

[9] N. Kulkarni, G.B. Lohiya, A Compact Microstrip Patch Antenna using Metamaterial, IJETT. 42 (2016) 365369

[10] H.P. Wang, S.Q. Feng, W.Y. Yang, H.P. Ma, G.H. Jia, S.Q. Xu, Sintering behavior and dielectric properties of $\mathrm{Al}_{2} \mathrm{O}_{3}$ ceramics with $\mathrm{CaMgSi}_{2} \mathrm{O}_{6}$ addition, J. Ceram. Soc. Jpn. 120 (2012) 268-271

[11] H. Kahari, M. Teirikangas, J. Juuti, H. Jantunen, Improvements and Modifications to Room-Temperature Fabrication Method for Dielectric $\mathrm{Li}_{2} \mathrm{MoO}_{4}$ Ceramics, J. Am. Ceram. Soc. 98 (2015) 687-689

[12] H. Kahari, P. Ramachandran, J. Juuti, H. Jantunen, Room-temperature-densified $\mathrm{Li}_{2} \mathrm{MoO}_{4}$ ceramic patch antenna and the effect of humidity, Int J Appl Ceram Technol. 14 (2017) 50-55

[13] J. Guo, A.L. Baker, H.Z. Guo, M. Lanagan, C.A. Randall, Cold sintering process: A new era for ceramic packaging and microwave device development, J. Am. Ceram. Soc. 100 (2017) 669-677

[14] D.W. Wang, D. Zhou, S.Y. Zhang, Y. Vardaxoglou, W.G. Whittow, D. Cadman, I.M. Reaney, ColdSintered Temperature Stable $\mathrm{Na}_{0.5} \mathrm{Bi}_{0.5} \mathrm{MoO}_{4}-\mathrm{Li}_{2} \mathrm{MoO}_{4}$ Microwave Composite Ceramics, ACS Sustainable Chemistry \& Engineering, 6 (2018) 2438-2444.

[15] F. Zuo, A. Badev, S. Saunier, D. Goeuriot, R. Heuguet, S. Marinel, Microwave versus conventional sintering: Estimate of the apparent activation energy for densification of $\alpha$-alumina and zinc oxide, J. Eur. Ceram. Soc. 34 (2014) 3103-3110

[16] R. Wroe, A.T. Rowley, Evidence for a non-thermal microwave effect in the sintering of partially stabilized zirconia, J. Mater. Sci. 31 (1996) 2019-2026.

[17] J. Wang, J. Binner, B. Vaidhyanathan, N. Joomun, J. Kilner, G. Dimitrakis, T.E. Cross, Evidence for the microwave effect during hybrid sintering, J. Am. Ceram. Soc. 89 (2006) 1977-1984

[18] D.A. Vinnik, A.Y. Tarasova, D.A. Zherebtsov, S.A. Gudkova, D.M. Galimov, V.E. Zhivulin, E.A. Trofimov, S. Nemrava, N.S. Perov, L.I. Isaenko, R. Niewa, Magnetic and Structural Properties of Barium Hexaferrite $\mathrm{BaFe}_{12} \mathrm{O}_{19}$ from Various Growth Techniques, Materials. 10 (2017) At. 10060578

[19] R. Nowosielski, R. Babilas, J. Wrona, Microstructure and magnetic properties of commercial barium ferrite powders, JAMME. 20 (2007) 307-310

[20] A. Bahadoor, Y. Wang, M.N. Afsar, Complex permittivity and permeability of barium and strontium ferrite powders in X, KU, and K-band frequency ranges, J. Appl. Phys. 97 (2005) At. 1853633

[21] K.K. Mallick, P. Shepherd, R.J. Green, Dielectric properties of M-type barium hexaferrite prepared by coprecipitation, J. Eur. Ceram. Soc. 27 (2007) 2045-2052

[22] S.V. Trukhanov, A.V. Trukhanov, V.G. Kostishyn, L.V. Panina, A.V. Trukhanov, V.A. Turchenko, D.I. Tishkevich, E.L. Trukhanova, O.S. Yakovenko, L.Y. Matzui, Investigation into the structural features and microwave absorption of doped barium hexaferrites, Dalton Trans. 46 (2017) 9010-9021

[23] X.G. Huang, J.A. Chen, L.X. Wang, Q.T. Zhang, Electromagnetic and microwave absorbing properties of W-type barium ferrite doped with $\mathrm{Gd}^{3+}$, Rare Met. 30 (2011) 44-48.

[24] W. Jing, Z. Hong, S.X. Bai, C. Ke, C.R. Zhang, Microwave absorbing properties of rare-earth elements substituted W-type barium ferrite, J. Magn. Magn. Mater. 312 (2007) 310-313.

[25] Y.P. Wu, C.K. Ong, Z.W. Li, L. Chen, G.Q. Lin, S.J. Wang, Microstructural and high-frequency magnetic characteristics of W-type barium ferrites doped with $\mathrm{V}_{2} \mathrm{O}_{5}$, J. Appl. Phys. 97 (2005) At.1861514

[26] W. Yuping, Development of Barium Hexaferrite composite materials for microwave absorption, Department of Physics, National University of Singapore, Singapore (2006)

[27] Z. Su, Development of low loss Hexaferrite materials for microwave applications, Department of Electrical and Computer Engineering, Northeastern University, Massachusetts, USA (2016)

[28] J.G. Fisher, H.Y. Sun, Y.G. Kook, J.S. Kim, P.G. Le, Growth of single crystals of $\mathrm{BaFe}_{12} \mathrm{O}_{19}$ by solid state crystal growth, J. Magn. Magn. Mater. 416 (2016) 384-390

[29] H. Kahari, M. Teirikangas, J. Juuti, H. Jantunen, Dielectric Properties of Lithium Molybdate Ceramic Fabricated at Room Temperature, J. Am. Ceram. Soc. 97 (2014) 3378-3379

[30] J.S. Dean, J.H. Harding, D.C. Sinclair, Simulation of Impedance Spectra for a Full Three- Dimensional Ceramic Microstructure Using a Finite Element Model, J. Am. Ceram. Soc. 97 (2014) 885-891

[31] C.H. Rycroft, VORO plus plus : A three-dimensional Voronoi cell library in C plus plus, Chaos. 19 (2009) At. 3215722 
[32] J.P. Heath, J. Dean, J. Harding, D. Sinclair, Simulation of impedance spectra for core-shell grain structures using finite element modeling, J. Am. Ceram. Soc. 98 (2015) 1925-1931

[33] M.M. Rahman, M.S. Sheak, S. Pervin, N. Uddin, F. Ahmed, M.A. Hossain, M. Rahaman, M.S. Bashar, T. Hossain, S. Akhter, Composition, Temperature and Frequency Dependent Magnetic, Dielectric and Electrical Properties of Magnesium-Zinc Ferrites, JBAS. 36 (2012) 199-212

[34] K. Singh, S.A. Band, W.K. Kinge, Effect of Sintering Temperature on Dielectric Properties of $\mathrm{Pb}\left(\mathrm{Fe}_{1 / 2} \mathrm{Nb}_{1 / 2}\right) \mathrm{O}_{3}$ Perovskite Material, Ferroelectrics, 306 (2004) 179-185 